\title{
Motion Detection by Background Subtraction Algorithm in FPGA
}

\author{
${ }^{1}$ Megha Mahesh Chakorkar, ${ }^{2}$ M.M. Patil \\ ${ }^{1,2}$ Department of Electronics and Telecommunication Sinhgad Academy of EngineeringPune, India
}

\begin{abstract}
In many applications moving object detection is important task. For detection of moving object this paper proposes a hardware solution based on background subtraction algorithm. It is implemented on FPGA.For this following are executed.

1)Image is captured by camera and stored in PC. 2)Both current and background images are sent to FPGA by serial communication 3).Background subtraction is done to obtain moving object.4)Position of moving object is located on FPGA.

Index Terms: background subtraction, motion detection
\end{abstract}

\section{Introduction}

The capability of extracting moving objects from a video sequence captured using a static camera is a typical first step in visual surveillance. The idea of background subtraction is to subtract or difference the current image from a reference background model. This paper proposes a method to detect moving object based on background subtraction. First of all, we find a background updating model based on statistical and use a dynamic optimization threshold method to obtain a more complete moving object.[1]

In the proposed system we use background subtraction method. Background subtraction method is general method of motion detection which uses the difference of the current image and the background image to detect moving objects. The key of this method is the initialization and update of background image and detection of moving object is also accurate.

The main target of motion detection process is to segment the foreground pixels that belong to the moving objects. To achieve this, there are several approaches for moving detection task, namely (1) the background subtraction, (2) the temporal difference of two successive frames and (3) the optical flow .

The background subtraction approach finds the moving objects by subtracting the current frame (pixel by pixel) from a reference image called background. Background can be found by means of an image selection process, which is executed during a initialization period .

On the other hand, the temporal difference of two successive frames has the same principle of the background subtraction method (subtraction of the current frame from a reference image), but in this case the reference image is the previous frame (in contrast with the background method, in which the reference image is fixed).

In the optical flow method the input to the method is an image or frame stream, $\mathrm{E}(\mathrm{x}, \mathrm{y}, \mathrm{t})$, where $\mathrm{x}$ and $y$ are the spatial coordinates of the stream in a time $t$. The value of the $E(x, y, t)$ function represents the brightness of the pixel $(x, y)$ in the image at time t. Therefore, the optical flow method is based on the principal that the object motion information is contained in the brightness changes of the image. It's main advantage is that it generates a complete vectorial field, which has a motion vector for each pixel.

All above described methods have good performance for motion detection problem. But optical flow is a very complex algorithm (it is necessary to store more than one image), requiring high memory resources. However, the background subtraction and the temporal difference are low cost algorithms. Temporal difference has problems for detecting the object's shape, generally, making difficult a posterior recognition stage. In the frame subtraction it is generally difficult to obtain a complete outline of a moving object, also the detection of moving object is not accurate .The disadvantage of optical flow method is that it is sensitive to noise and computational complexity is occurred. It is not suitable for real time demands. [2]

Therefore, in this paper a background subtraction algorithm for motion detection is presented due to its computational low cost, high performance and potential for other applications such as shape detection.

\section{Methodology:}

In this context, the moving object detection algorithm by background subtraction can be described as shown in Fig 1. 


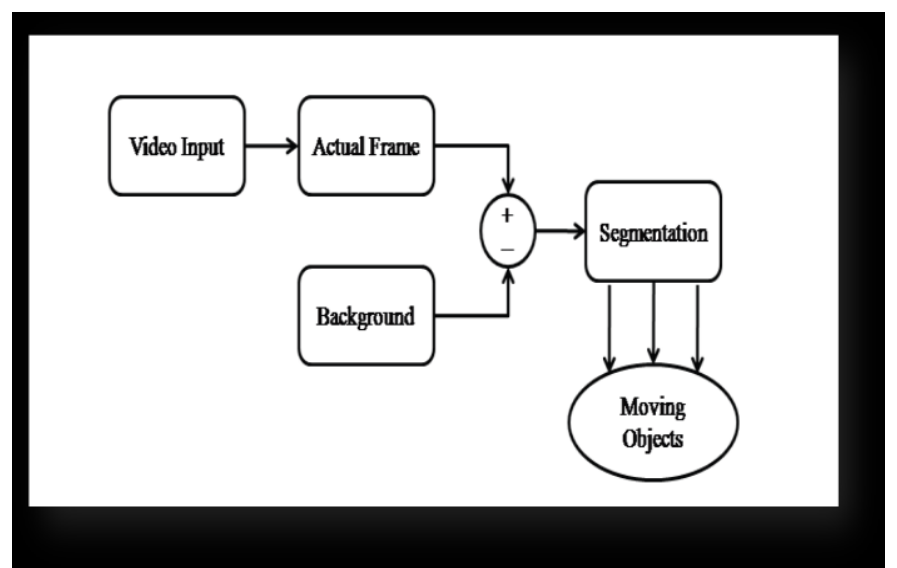

Figure1 The background subtraction algorithm for moving object detection

Each image of sequence is subtracted from background. The current frame can be used for background actualization (using a control bit). Afterward, the resulting image from the subtraction is segmented in order to produce a binary image that highlights the moving regions on the image. That moving regions belongs to the moving objects.[3]

Mathematically, the background subtraction algorithm can be defined by following equation ,

$$
\begin{array}{cc}
d(x, y, t)=1 & \text { if }|f(x, y, t)-B(x, y)|>T d \\
d(x, y, t)=0 & \text { otherwise }
\end{array}
$$

Where Td is a predetermined threshold, $\mathrm{f}(\mathrm{x}, \mathrm{y}, \mathrm{t})$ is an image taken at time $\mathrm{t}$ and $\mathrm{B}(\mathrm{x}, \mathrm{y})$ is the reference image (or background). In the dynamic image analysis, all pixels in the motion image $\mathrm{d}(\mathrm{x}, \mathrm{y}, \mathrm{t})$ with value " 1 " are Considered as moving objects in the scene.

Hardware setup:

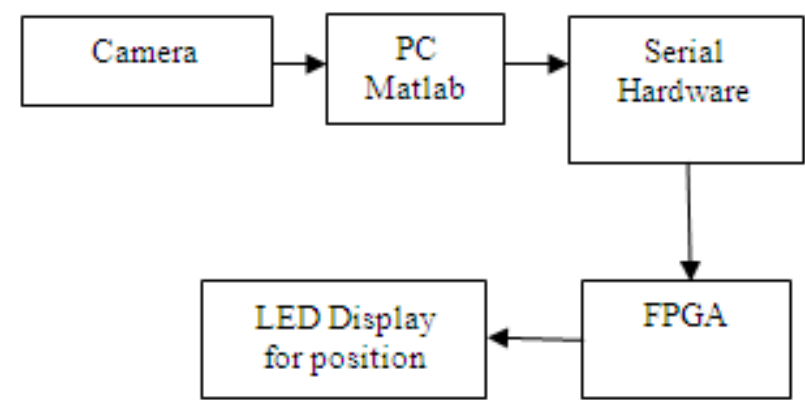

Fig 2: Block diagram of hardware setup

Software setup:

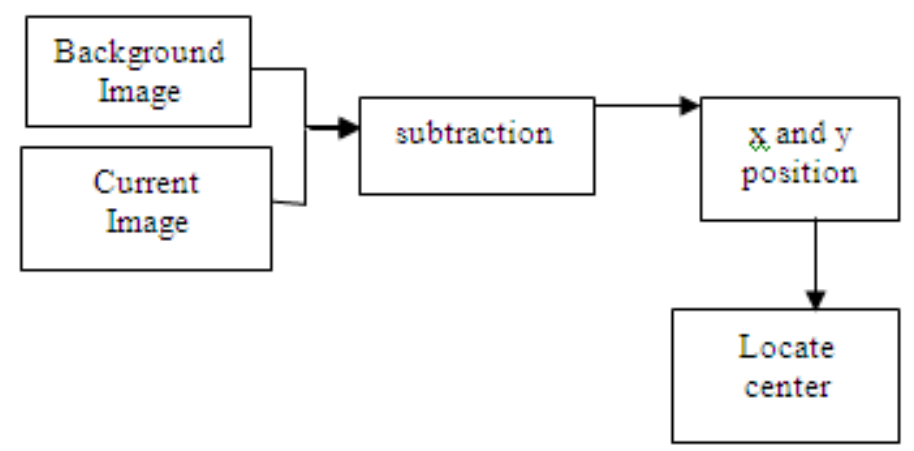

Figure 3 Block diagram of software setup 


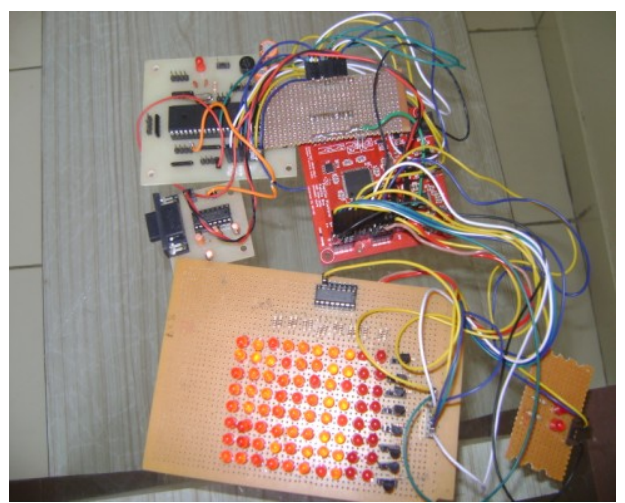

Figure 4 Hardware setup image 1

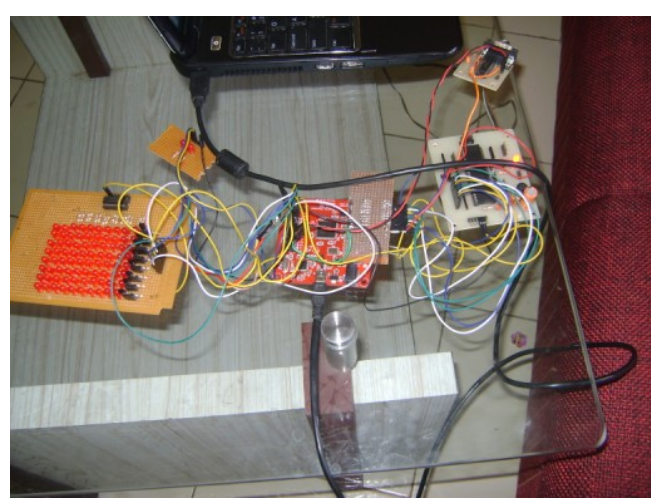

Figure 5: Hardware setup image2

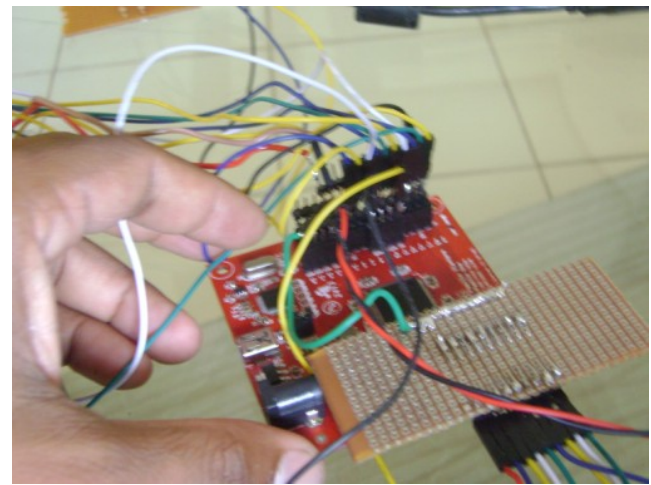

Figure 6: FPGA XC 3S 250e-4vq 100

\section{Features of FPGA board: XC 3S 250e-4vq 100:}

1. Fully assembled with a Xilinx Spartan $3 \mathrm{E}$ and $4 \mathrm{Mb}$ SP1 flash memory.

2. Provides an easy introduction to FPGA, digital electronics \& system on chip.design

3. Two channel USB connection for JTAG and serial communications

4. 4 independent power rails at $5 \mathrm{v}, 3.3 \mathrm{v}$ and $1.2 \mathrm{v}$

5. Input voltage $6.5-15 \mathrm{v}$

6. $48 \mathrm{I} / \mathrm{O}$ lines

\section{Results:} MATLAB.

In this case the image capturing is done by USB camera connected to PC. Image is send to FPGA by In MATLAB trigger is set and firstly when gets trigger the first image is stored as background and all other images are send to FPGA for subtraction algorithm.

XC3S 250 Spartan3 board is used as FPGA.

For software development Matlab 2012 and Xilinx ISE are used. 
Moving object detected can be seen on Matlab as images and position of moving object can be seen as LEDs glows on the FPGA.

Results obtained by software tool:

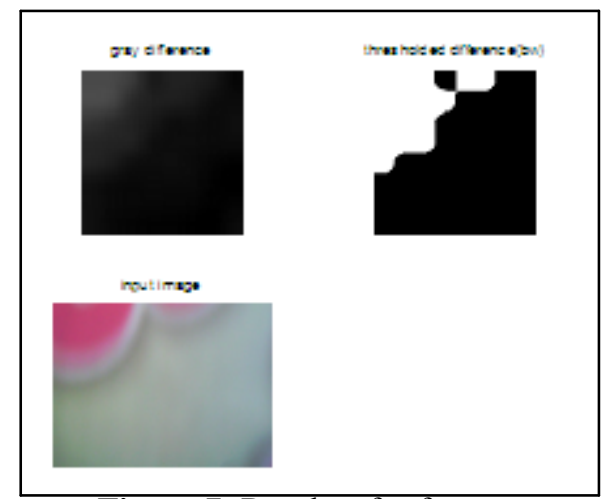

Figure 7: Results of software

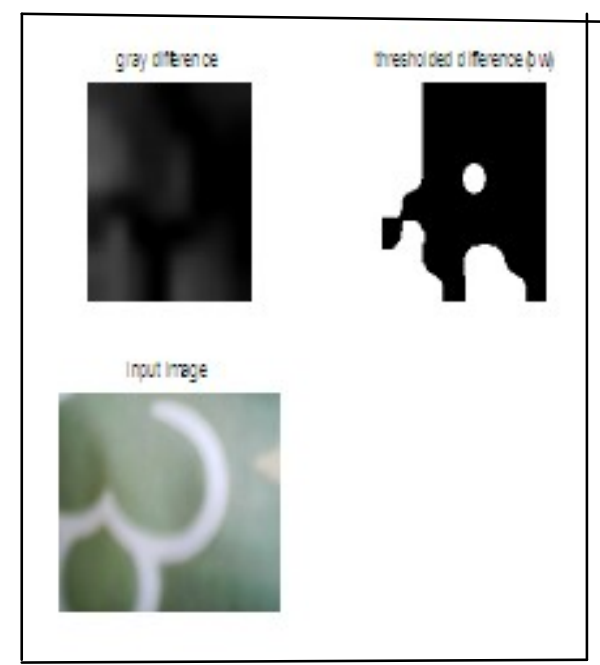

Figure 8: Result of software

\section{Conclusion:} developed.

In this work a moving object motion detection system based on background subtraction algorithm was

Additionally, the system is capable to detect an object by extracting its shape and calculating the centre. The object position is send to a PC or another platform via RS-232 interface.

\section{References:}

[1]. C. Sanchez-Ferreira, J.Y.Mori, and C.H.Lianos, "Background Subtraction Algorithm for Moving Object detection in FPGA" , Programmable Logic(SPL),2012 viii southern conference on digital object Identifier:10:1109/SPL.2012,P-P 1-6

[2]. Marek Wójcikowski,Robert Zaglewski,and Bogdan Pankiewicz "FPGA-Based Real-Time Implementation of Detection algorithm for Automatic Traffic Surveillance Sensor etwork"

[3]. R. Hartenstein, "Why we need reconfigurable computing education",1 1st International Workshop on Reconfigurable Computing Education,WRCE2006, Germany, 2006.

[4]. Y. Zhou and Y. Tan, Gpu-based parallel particle swarm optimization,Proc. IEEE Int. Congress on Evolutionary Computation, pp. $1493-1500,2009$.

[5]. L. Veronese and R. Krohling, Swarms's flight: Accelerating the particlesusing c-cuda, Proc. IEEE Int. Congress on Evolutionary Computation,pp. 3264-3270, Norway, 2009.

[6]. D. Mu noz, C. Llanos, L. Coelho, and M. Ayala-Rinc'on, Comparison between two FPGA implementation of the particle swarm optimization algorithm for high-performance embedded applications, The IEEE Fifth International Conference on Bio-Inspired Computing: Theories and Applications (BIC-TA 2010), vol. 1, pp. 1637-1645, 2010.

[7]. J. Y. Mori, C. S'anchez-Ferreira, D. Mũnoz, C. Llanos and P. Berger, An Unified Approach for Convolution-Based Image Filtering on Reconfigurable Systems, Proc. IEEE VII Southern Conference on Programmable Logic (SPL), pp. 63-68, C'ordoba, Argentina, 2011. 